\title{
Virtual Research Environment
}

Leonardo Candela ${ }^{1}$

1 Italian National Research Council

\section{Source}

Leonardo Candela, Donatella Castelli, Pasquale Pagano. (2013). Virtual Research

Environments: An Overview and a Research Agenda. Data Sci. J., vol. 12 (0), GRDI75-

GRDI81.

A web-based, community-oriented, comprehensive, flexible, and secure working environments conceived to serve the needs of modern science. 